\title{
COPRODUCTS OF KLEENE ALGEBRAS
}

\author{
WILLIAM H. CORNISH and PETER R. FOWLER \\ (Received 19 October 1976; revised 14 February 1977, 1 May 1978) \\ Communicated by H. Lausch
}

\begin{abstract}
The coproduct of a family of Kleene algebras is determined firstly by describing the maximal homomorphic image of a De Morgan algebra in the subvariety of Kleene algebras and, secondly, by characterizing the categorical product in the dual category of Kleene spaces.
\end{abstract}

Subject classification (Amer. Math. Soc. (MOS) 1970): 02 J 05, 06 A 35.

\section{Introduction}

A De Morgan algebra $(L ; \vee, \wedge, \sim, 0,1)$ is an algebra of type $\langle 2,2,1,0,0\rangle$ such that $(L ; \vee, \wedge, 0,1)$ is a bounded distributive lattice with largest element 1 , smallest element 0 , and an involutorial dual order-isomorphism $\sim$, that is, the equations $\sim \sim x=x, \sim(x \vee y)=\sim x \wedge \sim y, \sim(x \wedge y)=\sim x \vee \sim y, \sim 0=1$ and $\sim 1=0$ are identically satisfied. A Kleene algebra is a De Morgan algebra which also satisfies the equation $(x \wedge \sim x) \wedge(y \vee \sim y)=x \wedge \sim x$, while a Boolean algebra is a De Morgan algebra satisfying the identity $x \wedge \sim x=0$. The varietal categories of De Morgan, Kleene and Boolean algebras are denoted by $\mathbf{M}, \mathbf{K}$ and $\mathbf{B}$, respectively, and $M \supset K \supset B$. Each of these categories can be considered as a subcategory of the category $D$ of bounded distributive lattices and $(0,1)$-preserving lattice-homomorphisms. It will of ten be desirable to emphasize the preservation of the $\sim$-operation by homomorphisms, congruences and subalgebras and so we then speak of $\sim$ homomorphisms, $\sim$-congruences and $\sim$-subalgebras.

If $\mathrm{X}$ is any one of the above categories and $\left\{Y_{i}\right\}$ is a family of objects in $\mathrm{X}$ then their x-coproduct is denoted by $\coprod_{\mathrm{x}} Y_{i}$; the coproduct of two objects $Y, Z$ in $\mathrm{x}$ is denoted by $Y \coprod_{\mathrm{x}} Z$. Coproducts in $\mathrm{D}$ have been described, in detail, by Grätzer (1971), Section 12, pp. 128-137, and Balbes and Dwinger (1974), Chapter 7, pp. 132-150. On the other hand, Balbes and Dwinger (1974) gave, in Theorem 2, 
p. 216, a result of Berman and Dwinger which asserts that the M-coproduct of a family of objects $\left\{Y_{i}\right\}$ in $\mathrm{M}$ is actually their D-coproduct together with the $\sim$-operation which is the unique extension to $\coprod_{\mathrm{D}} Y_{i}$ of the $\sim$-operation on each of the $Y_{i}$. Using a representation of the dual of $M$, Cornish and Fowler (1977) gave an alternative proof of this theorem and also a few results on $\mathrm{K}$-coproducts, the principal being the result that the $\mathrm{M}$ - and $\mathrm{K}$-coproducts of a family of Kleene algebras are $\sim$-isomorphic if and only if all, but possibly one, of the algebras is actually Boolean. Thus, the purpose of this paper is to describe the K-coproduct of a family of arbinary Kleene algebras. In Section 1, we describe the smallest $\sim$-congruence on a De Morgan algebra such that the quotient algebra is a Kleene algebra; there it is interesting to contrast our description with that of the smallest $\sim$-congruence such that the quotient algebra is a Boolean algebra. In Section 2, we describe the dual of the various objects appearing in Section 1 and as a consequence obtain the product in the category of Kleene spaces; the necessary topological preliminaries are included at the start of Section 2. In the final section we give some results on free Kleene algebras. Free Kleene algebras have also been discussed by Berman and Dwinger (1973) and Berman and Köhler (1976).

We would like to thank the referees for their comments and for bringing to our attention the aforementioned two papers together with the preprint by Urquhart, which contain some overlap with our presentation.

\section{Maximal homomorphic images}

We begin this section with a part of the folk-lore of Universal Algebra. Let w be a subvariety of a variety $\mathrm{v}$ of finitary algebras. Suppose $A$ is an algebra in $\mathrm{v}$, whose congruence-lattice is $C(A)$. As varieties are closed under the formation of subdirect products, the set $Y=\{\Theta \in C(A): A / \Theta \in \mathrm{W}\}$ has a smallest member, namely $\Theta_{\mathrm{w}}(A)=\bigcap\{\Theta: \Theta \in Y\}$. The quotient algebra $A / \Theta_{\mathrm{w}}(A)$ is called the maximal homomorphic image of $A$ in w since each homomorphic image of $A$, which is in $\mathrm{w}$, is itself a homomorphic image of this algebra. Better still, as varieties are closed under the formation of subalgebras, each v-homomorphism $f$ of $A$ into a W-algebra $B$ can be factored uniquely into the form $f=f \circ t$, where $t$ is the canonical homomorphism of $A$ onto $A / \Theta_{\mathrm{w}}(A)$ and $\bar{f}$ is a homomorphism of $A / \Theta_{\mathrm{w}}(A)$ into $B$. Thus, we obtain a reflector or reflective functor mapping the varietal category $\mathrm{v}$ into the subcategory $\mathrm{w}$. But a reflector preserves coproducts, see Balbes and Dwinger (1974), Theorem 5, p. 29. In other words, if $\left\{B_{i}\right\}$ is a family of w-algebras then $\coprod_{\mathrm{w}} B_{i}$ is isomorphic to $\coprod_{\mathrm{v}} B_{i} / \Theta_{\mathrm{w}}\left(\coprod_{\mathrm{v}} B_{i}\right)$.

Taking $\mathrm{v}$ to be the variety $\mathrm{M}$ of De Morgan algebras, $\mathrm{w}$ to be the subvariety $\mathrm{K}$ of Kleene algebras and using the notation $\kappa(A)$ in place of $\Theta_{\mathrm{w}}(A)$ for any $A \in \mathrm{V}=\mathrm{M}$, we can summarize our observations in the following result. 
Proposition 1.1. Let $\left\{K_{i}\right\}$ be a family of Kleene algebras. Then $\coprod_{\mathrm{K}} K_{i}$ is isomorphic to $\coprod_{\mathrm{M}} K_{i} / \kappa\left(\coprod_{\mathrm{M}} K_{i}\right)$.

Thus, in a sense we will have described $\mathrm{K}$-coproducts if we give a description of the congruence $\kappa(A)$ on any Kleene algebra $A$. Of course, the B-coproduct of Boolean algebras is well known. Nevertheless, it is not without interest to describe the congruence $\beta(A)$, corresponding to the case where $A \in \mathrm{V}=\mathrm{M}$ and $\mathrm{W}=\mathrm{B}$.

Let $Y$ be a non-empty subset of a De Morgan algebra $L$. Then, we use the following notation: $\sim Y=\{\sim y: y \in Y\},(Y]$ is the ideal of the underlying lattice which is generated by $Y$, and $[Y]$ is the filter of the underlying lattice generated by $Y$. In general, $\sim(Y]=[\sim Y)$ and $\sim[Y)=(\sim Y]$ so that $Y$ is an ideal if and only if $\sim Y$ is a filter. A particularly interesting subset is $T=\{t \in L: t \leqslant \sim t\}=\{x \wedge \sim x: x \in L\}$. We define the nucleus $n(L)$ of a De Morgan algebra $L$ to be the ideal $(T]$. As $T$ is hereditary, $n(L)=\left\{t_{1} \vee \ldots \vee t_{n} t_{i} \in T\right.$ for $\left.1 \leqslant i \leqslant n\right\}$. In view of the remarks in Section 4 of the paper by Kalman (1958), the following result is given without proof.

Proposition 1.2. The following conditions on a De Morgan algebra $L$ are equivalent.

(i) $L$ is a Kleene algebra.

(ii) For any $x, y \in L$, there exists $z \in L$ such that $(x \wedge \sim x) \vee(y \wedge \sim y)=(z \wedge \sim z)$.

(iii) $n(L)=\{x \wedge \sim x: x \in L\}$.

(iv) $\{x \vee \sim x: x \in L\}$ is a filter.

Let $J$ and $F$ be an ideal and a filter, respectively, in a distributive lattice. Then, the smallest congruences $\Theta(J)$ and $\Theta(F)$ which have $J$ and $F$, respectively, as congruence classes are given by

and

$$
x \equiv y(\Theta(J))(x, y \in L) \Leftrightarrow x \vee j=y \vee j \text { for some } j \in J
$$

$$
x \equiv y(\Theta(F))(x, y \in L) \Leftrightarrow x \wedge f=y \wedge f \text { for some } f \in F,
$$

compare with Grätzer (1971), Corollary 4, p. 88, or Balbes and Dwinger (1974), Theorem 17, p. 60.

In the following theorem, the notation and lattice operations are understood to refer to the lattice of lattice-congruences of the underlying lattice of a given De Morgan algebra.

THEOREM 1.3. Let L be a De Morgan algebra. Then,

(i) $\kappa(L)=\Theta(n(L)) \cap \Theta(\sim n(L))$, and

(ii) $\beta(L)=\Theta(n(L)) \vee \Theta(\sim n(L))$.

Proof (i) It is easily shown that $\Theta(n(L)) \cap \Theta(\sim n(L))$ has the substitution property for $\sim$ and so it is a $\sim$-congruence. Let $x, y \in L$. Then,

$$
(x \wedge \sim x) \vee(y \vee \sim y) \equiv 0 \equiv x \wedge \sim x(\Theta(n(L)))
$$


and

$$
(x \wedge \sim x) \wedge(y \vee \sim y) \equiv(x \wedge \sim x) \wedge 1 \equiv x \wedge \sim x(\Theta(\sim n(L))) .
$$

Hence,

$$
(x \wedge \sim x) \vee(y \vee \sim y) \equiv x \wedge \sim x(\Theta(n(L)) \cap \Theta(\sim n(L))),
$$

and consequently $L / \Theta(n(L)) \cap \Theta(\sim n(L))$ is a Kleene algebra.

Let $\Phi$ be a $\sim$-congruence on $L$ such that $L / \Phi$ is a Kleene algebra. Let $a, b \in L$ be such that $a \equiv b(\Theta(n(L)) \cap \Theta(\sim n(L)))$. Then there exist $x_{1}, \ldots, x_{m}, y_{1}, \ldots, y_{n} \in L$ such that

$$
a \vee\left(x_{1} \wedge \sim x_{1}\right) \vee \ldots \vee\left(x_{m} \wedge \sim x_{m}\right)=b \vee\left(x_{1} \wedge \sim x_{1}\right) \vee \ldots \vee\left(x_{m} \wedge \sim x_{m}\right)
$$

and

$$
a \wedge\left(y_{1} \vee \sim y_{1}\right) \wedge \ldots \wedge\left(y_{n} \vee \sim y_{n}\right)=b \wedge\left(y_{1} \vee \sim y_{1}\right) \wedge \ldots \wedge\left(y_{n} \vee \sim y_{n}\right) .
$$

Let $\bar{w}$ denote the $\Phi$-class of any $w \in L$. As $L / \Phi$ is a Kleene algebra $\bar{x}_{i} \wedge \sim \bar{x}_{i} \leqslant \bar{y}_{j} \vee \sim \bar{y}_{j}$ for any $i=1, \ldots, m$ and $j=1, \ldots, n$. It follows that

$$
\left.\bar{a} \vee\left[\left(\bar{x}_{1} \wedge \sim \bar{x}_{1}\right) \vee \ldots \vee\left(\bar{x}_{m} \wedge \sim \bar{x}_{m}\right)\right]=\bar{b} \vee\left[\bar{x}_{1} \wedge \sim \bar{x}_{1}\right) \vee \ldots \vee\left(\bar{x}_{m} \wedge \sim \bar{x}_{m}\right)\right]
$$

and

$$
\bar{a} \wedge\left[\left(\bar{x}_{1} \wedge \sim \bar{x}_{1}\right) \vee \ldots \vee\left(\bar{x}_{m} \wedge \sim \bar{x}_{m}\right)\right]=\bar{b} \wedge\left[\left(\bar{x}_{1} \wedge \sim \bar{x}_{1}\right) \vee \ldots \vee\left(\bar{x}_{m} \wedge \sim \bar{x}_{m}\right)\right] .
$$

As $L / \Theta$ is distributive it follows that $\bar{a}=\bar{b}$ and so $\Theta(n(L)) \cap \Theta(\sim n(L)) \subseteq \Phi$. Thus (i) is established.

(ii) It is readily shown that $\Theta(n(L)) \vee \Theta(\sim n(L))$ is a $\sim$-congruence and, as $x \wedge \sim x \equiv 0(\Theta(n(L))), x \wedge \sim x \equiv 0(\Theta(n(L)) \vee \Theta(\sim n(L)))$ for any $x \in L$, the associated quotient algebra is Boolean. Since $0 \equiv x \wedge \sim x$ and $1 \equiv x \vee \sim x$ modulo $\beta(L)$ it follows that $\Theta(n(L)), \Theta(\sim n(L)) \subseteq \beta(L)$. It should now be apparent that

$$
\beta(L)=\Theta(n(L)) \vee \Theta(\sim n(L)) .
$$

It should be noted that in their Theorem 4.3, Berman and Dwinger (1973) described the congruence $\beta(L)$ as the smallest $\sim$-congruence containing

$$
\{x \wedge \sim x: x \in L\}
$$

in a single congruence-class.

THEOREM 1.4. Let $\left\{L_{i}\right\}$ be a family of De Morgan algebras. Let $L=\bigsqcup_{\mathrm{M}} L_{i}$ and regard each $L_{i}$ as a subalgebra of $L$. Then,

(i) $n(L)=\vee\left(n\left(L_{i}\right)\right]_{\mathrm{L}}$, where $\left(n\left(L_{i}\right)\right]_{\mathrm{L}}$ is the ideal of $L$ generated by $n\left(L_{i}\right)$ and the join is taken within the lattice of ideals of $L$, and

(ii) $\sim n(L)=\vee\left[\sim n\left(L_{i}\right)\right)_{\mathrm{L}}$, where $\left[\sim n\left(L_{i}\right)\right)_{\mathrm{L}}$ is the filter of $L$ generated by $\sim n\left(L_{i}\right)$ and the join is taken within the lattice of filters of $L$. 
Proof. Obviously each $n\left(L_{i}\right)$ is contained within $n(L)$ and so $\vee\left(n\left(L_{i}\right)\right]_{\mathrm{L}} \subseteq n(L)$.

Let $a \in L$ be a generator of $n(L)$, so that $a \leqslant \sim a$. Of course, the $L_{i}$ generate $L$ as a distributive lattice and so

$$
a=\bigvee_{r=1}^{s} a_{r_{1}} \wedge \ldots \wedge a_{r_{k}},
$$

where for all $1 \leqslant r \leqslant s$ and $1 \leqslant j \leqslant k, a_{r_{j}} \in L_{j}$ and $L_{1}, \ldots, L_{k} \in\left\{L_{i}\right\}$. Then,

$$
\sim a=\bigwedge_{r=1}^{s} \sim a_{r_{1}} \vee \ldots \vee \sim a_{r_{k}}
$$

and as $a \leqslant \sim a$, we have for each $1 \leqslant r \leqslant s$,

$$
a_{r_{1}} \wedge \ldots \wedge a_{r_{k}} \leqslant \sim a_{r_{1}} \vee \ldots \vee \sim a_{r_{k}}
$$

But by the result of Berman and Dwinger referred to in the Introduction, $L$, as a distributive lattice, is isomorphic to $\coprod_{\mathrm{D}} L_{i}$. Hence; by Grätzer (1971), Theorem 5 , p. 131, there exists $1 \leqslant j_{r} \leqslant k$ such that $a_{r_{j r}} \leqslant \sim a_{r_{j r}}$, and so $a_{r_{r}} \in n\left(L_{j r}\right)$. It follows that $a \in \vee\left(n\left(L_{i}\right)\right]_{\mathrm{L}}$. Thus, (i) is established; the proof of (ii) proceeds in a dual fashion.

Thus, Theorems 1.3 and 1.4 give detailed information about the description of the $\mathrm{K}$-coproduct, as given in 1.1. We now consider this description in a few particular cases.

LEMMA 1.5. Let $a$ and $b$ be elements of $a$ bounded distributive lattice L. Then, $\Theta(0, a) \cap \Theta(b, 1)=\Theta(a \wedge b, a)$, where for any $x, y \in L, \Theta(x, y)$ denotes the smallest congruence identifying $x$ and $y$.

Proof. By Theorem 3, p. 87 of Grätzer (1971) and the distributivity of the congruence lattice of a lattice (Theorem 11, p. 93 of Grätzer (1971)),

$$
\begin{aligned}
\Theta(a \wedge b, a) & =\Theta(a \wedge b, 1) \cap \Theta(0, a)=(\Theta(a, 1) \vee \Theta(b, 1)) \cap \Theta(0, a) \\
& =(\Theta(a, 1) \cap \Theta(0, a)) \vee(\Theta(0, a) \cap \Theta(b, 1)) \\
& =\omega \vee(\Theta(0, a) \cap \Theta(b, 1))=\Theta(0, a) \cap \Theta(b, 1) .
\end{aligned}
$$

where, as is usual, $\omega$ denotes the equality congruence.

THeOREM 1.6. Let $K_{1}, \ldots, K_{t}$ be Kleene algebras. Suppose for each $1 \leqslant r \leqslant t, n\left(K_{r}\right)$ is a principal ideal, whereby $n\left(K_{r}\right)=\left\{a \in K_{r}: a \leqslant x_{r} \wedge \sim x_{r}\right\}$ for a suitable choice of $x_{r} \in K_{r}$. Then,

$$
\kappa\left(\bigsqcup_{r=1}^{t} K_{r}\right)=\Theta\left(\bigvee_{r=1}^{t}\left(x_{r} \wedge \sim x_{r}\right) \wedge \bigwedge_{r=1}^{t}\left(x_{r} \vee \sim x_{r}\right), \bigvee_{r=1}^{t}\left(x_{r} \wedge \sim x_{r}\right)\right)
$$


PROOF. The theorem is a combination of our previous results.

An element $x$ of a Kleene algebra $L$ is called a fixed point if $x=\sim x$. It is easy to see that a Kleene algebra can have at most one fixed point and that, when one exists, it is the generator of the nucleus.

COROLlaRY 1.7. Let $K_{1}, \ldots, K_{t}$ be Kleene algebras with respective fixed points $y_{1}, \ldots, y_{t}$. Then,

$$
\kappa\left(\bigsqcup_{r=1}^{t} K_{r}\right)=\Theta\left(\bigwedge_{r=1}^{t} y_{r}, \bigvee_{r=1}^{t} y_{r}\right)
$$

the smallest $(\sim-)$ congruence identifying $y_{1}, y_{2}, \ldots, y_{t}$.

To illustrate 1.7 let us consider the coproduct $3 \bigsqcup_{\mathrm{K}} 3$, where 3 is the 3-element Kleene algebra $\{0, a, 1: 0<a<1, a=\sim a\}$. Then, as a lattice $3 \bigsqcup_{\mathrm{M}} 3$ is isomorphic to $3 \bigsqcup_{D} 3$, and it has the induced involution so we can describe the $\mathrm{M}$-coproduct of two copies of 3 by the set

$\{0, a \wedge b, a, b, a \vee b, 1: 0<a \wedge b<a, b<a \vee b<1 ; a=\sim a, b=\sim b$, and so on $\}$.

Using 1.1 and 1.7 we conclude that $3 \coprod_{\mathrm{K}} 3=3$. For other related results, see Section 3 of this paper.

Let $\mathrm{X}$ be any one of $\mathrm{M}, \mathrm{K}$ and $\mathrm{D}$ and $F_{\mathrm{X}}\left(x_{1}, \ldots, x_{t}\right)$ denote the free $\mathrm{X}$-algebra whose free generators are $x_{1}, \ldots, x_{t}$. Of course, this free algebra is isomorphic to $t$ copies of the free X-algebra on one generator. The free algebras $F_{\mathrm{M}}\left(x_{1}\right)$ and $F_{\mathrm{K}}\left(x_{1}\right)$ are one and the same; as lattices they coincide with the 6-element lattice $F_{\mathrm{D}}\left(x_{1}, \sim x_{1}\right)$, where $x_{1}$ and $\sim x_{1}$ are the free generators, and the involution is the unique extension of the map $x_{1} \leftrightarrow \sim x_{1}, \sim x_{1} \rightarrow x_{1}$. Moreover, $n\left(F_{\mathrm{K}}\left(x_{1}\right)\right)=\left\{0, x_{1} \wedge \sim x_{1}\right\}$. In general, $F_{\mathrm{M}}\left(x_{1}, \ldots, x_{t}\right)$ is as a lattice the free D-algebra on the $2 t$ free generators $x_{1}, \ldots, x_{t}, \sim x_{1}, \ldots, \sim x_{t}$, and the involution is the unique extension of the map $x_{i} \leftrightarrow \sim x_{i}, \sim x_{i} \rightarrow x_{i}$ for all $1 \leqslant i \leqslant t$. These remarks are contained in Theorem 3, p. 218 of Balbes and Dwinger (1974). Using 1.1 and 1.6 and these observations, we obtain a description of the free Kleene algebra on a finite number of generators which can be symbolized by

COROLlary 1.8 .

$$
F_{\mathrm{K}}\left(x_{1}, \ldots, x_{t}\right) \cong \frac{F_{\mathrm{D}}\left(x_{1}, \ldots, x_{t}, \sim x_{1}, \ldots, \sim x_{t}\right)}{\Theta\left(\bigvee_{r=1}^{i}\left(x_{r} \wedge \sim x_{r}\right) \wedge \bigwedge_{r=1}^{t}\left(x_{r} \vee \sim x_{r}\right), \bigvee_{r=1}^{t}\left(x_{r} \wedge \sim x_{r}\right)\right)} .
$$

\section{Dual spaces}

We now consider the topological representations of the duals of $M$ and $K$ and so develop an effective method for obtaining $\mathrm{K}$-coproducts. 
An ordered (topological) space $(X ; \leqslant, \tau)$ is a partially ordered set $(X ; \leqslant)$ which has a topology $\tau$; when there is no possibility of confusion we simply refer to an ordered topological space $(X ; \leqslant, \tau)$ by means of the underlying set $X$. A subset $U$ of an ordered space $X$ is called increasing (decreasing) if $x \leqslant y, x, y \in X$, and $x \in U(y \in U)$ imply $y \in U(x \in U)$. A totally order-disconnected space $X$ is an ordered space such that whenever $x \leqslant y, x, y \in X$, there exists a clopen (= closed and open) increasing subset $U$ such that $x \in U$ whilst $y \in X \backslash U$. The set $\mathscr{D}(X)$ of all clopen increasing subsets of an ordered space $X$ is always a bounded distributive lattice with respect to the set-theoretic operations. Moreover, if $X$ is compact and totally order-disconnected then $\mathscr{D}(X) \cup\{X \backslash U: U \in \mathscr{D}(X)\}$ is a sub-base for the topology on $X$. The category, whose objects are compact totally order-disconnected spaces and whose morphisms are continuous monotone (= order-preserving) functions, is denoted by roDc. It is well known that TODC and D are dual isomorphic as categories, see, for example, Cornish (1975). The dual of a D-object $L$ is $\operatorname{Pr}(L)$, the Priestley space of $L ; \operatorname{Pr}(L)$ can be considered as the set of all prime ideals of $L$, ordered by the reverse of set-inclusion, and the map $a \leftrightarrow(p \in \operatorname{Pr}(L): a \notin p\}, a \in L$, is a lattice-isomorphism of $L$ onto $\mathscr{D}(\operatorname{Pr}(L))$.

Cornish and Fowler (1977) showed that the dual of the category $M$ is isomorphic to a category whose objects $(X, g)$ are totally order-disconnected spaces $X$ which possess a homeomorphism $g: X \rightarrow X$ such that $g$ is also a dual order-isomorphism and $g^{2}=1_{X}$, and whose morphisms are continuous monotone functions $f:\left(X_{1}, g_{1}\right) \rightarrow\left(X_{2}, g_{2}\right)$ such that $f \circ g_{1}=g_{2} \circ f$. Previously, we called this category G-TODC. However, in this paper we will call it the category of De Morgan spaces and denote it by Ms. If $L$ is an object in $\mathrm{M}$ then its dual object in $\operatorname{Ms}$ is $(\operatorname{Pr}(L), g)$, where $g$ is defined by $g(p)=L \mid \sim p$ for each prime ideal $p \in \operatorname{Pr}(L)$; if $\sim$ is defined on $\mathscr{D}(\operatorname{Pr}(L))$ by $\sim U=\operatorname{Pr}(L) \backslash g(U), U \in \mathscr{D}(\operatorname{Pr}(L))$ then $L$ and $\mathscr{D}(\operatorname{Pr}(L))$ are $\sim$-isomorphic De Morgan algebras with respect to the canonical map $a \rightarrow\{p \in \operatorname{Pr}(L): a \notin p\}$. The category Ks of Kleene spaces is the full subcategory of ms whose objects $(X, g)$ are such that $x$ and $g(x)$ are comparable for each $x \in X$; in Theorem 3.2, Cornish and Fowler (1977) showed that $\mathrm{K}$ and $\mathrm{Ks}$ are dual isomorphic categories.

An order-subspace $Y$ of an ordered space $X$ is subspace $Y$ of the topological space $X$, endowed with the partial order which is the restriction of the order on $X$ to $Y$. A subset $Y$ of a De Morgan space $(X, g)$ is said to be $g$-invariant if $g(Y) \subseteq Y$. As $g$ is an involution, a subset $Y$ is $g$-invariant if and only if $g(Y)=Y$.

Theorem 8.3 of Hashimoto (1952) may be interpreted in the context of the Priestley duality. It yields a dual-order isomorphism $T \rightarrow \Theta(T)$ of the lattice of closed order-subspaces $T$ of the Priestley space of the bounded distributive lattice $L$ onto the lattice of congruences of $L ; \Theta(T)$ is the congruence given by: $a \equiv b(\Theta(T)), a, b \in L$, if and only if $\hat{a} \cap T=\hat{b} \cap T$, where $\hat{a}=\{P \in \operatorname{Pr}(L): a \notin P\}$. When $L$ is a De Morgan algebra and $T$ is in addition $g$-invariant, it is not hard to see that $\Theta(T)$ is a $\sim$-congruence and $T$, together with the restriction of $g$, is isomorphic to 
the De Morgan space of the algebra $L / \Theta(T)$. Thus, for any De Morgan algebra $L$, Hashimoto's map $T \leftrightarrow \Theta(T)$, induces a dual-order isomorphism of the lattice of g-invariant closed order-subspaces of De Morgan space $(\operatorname{Pr}(L), g)$ onto the lattice of -congruences of $L$. In Theorem 5, Urquhart (1978) gave an ab initio proof of a similar result for algebras in which all the De Morgan axioms hold with possible exception of the law of double negation.

We now define some order-subspaces of a De Morgan space $(X, g)$. They are:

$$
\begin{aligned}
\vec{X} & =\{x \in X: x \geqslant g(x)\}, \\
\bar{X} & =\{x \in X: x \leqslant g(x)\} \\
X_{\mathbf{K}} & =\vec{X} \cup \bar{X}
\end{aligned}
$$

and

$$
X_{\mathbf{B}}=\vec{X} \cap \overleftarrow{X} .
$$

Of course, $g(\vec{X})=\bar{X}, g(\bar{X})=\vec{X}$ and so both $X_{\mathrm{K}}$ and $X_{\mathrm{B}}$ are $g$-invariant.

Lemma 2.1. Let $(X, g)$ be a De Morgan space. Then, each of $\vec{X}, \bar{X}, X_{\mathbf{K}}$ and $X_{\mathrm{B}}$ is closed.

Proof Suppose $x \notin \vec{X}$ so that $x \neq g(x)$. Let $U \in \mathscr{D}(X)$ be such that $g(x) \in U$ and $x \in X \backslash U$. Then, $g(U) \cap(X \backslash U)$ is a clopen decreasing neighbourhood of $x$ which is readily seen to be disjoint from $\vec{X}$. Thus, $\vec{X}$ is closed. As $g$ is a homeomorphism $\bar{X}=g(\vec{X})$ is closed and the result follows.

TheOREM 2.2. Let $L$ be a De Morgan algebra with associated De Morgan space $\left(X=P_{r}(L), g\right)$. Then,

(i) $\bar{X}$ is the dual in ToDc of the lattice $L / \Theta(n(L))$.

(ii) $\vec{X}$ is the dual in ToDc of the lattice $L / \Theta(\sim n(L))$.

(iii) $\left(X_{\mathrm{K}}, g \mid X_{\mathrm{K}}\right)$ is the dual in MS of the Kleene algebra $L / \kappa(L)$.

(iv) $\left(X_{\mathrm{B}}, g \mid X_{\mathrm{B}}\right)$ is the dual in MS of the Boolean algebra $L / \beta(L)$.

Proof. (i) Let $a \in L$. Suppose $p \in(a \wedge \sim a)^{\wedge} \cap \bar{X}$. Then, $p \in(a \wedge \sim a)^{\wedge}$ and $p \leqslant g(p)$. As $(a \wedge \sim a)^{\wedge}$ is increasing $g(p) \in(a \wedge \sim a)^{\wedge}$, that is, $a \wedge \sim a \notin g(p)$. But $p \in(a \wedge \sim a)^{\wedge}$ and so $a \wedge \sim a \notin p$. Thus, $a \vee \sim a \notin \sim p$ and hence $a \vee \sim a \in g(p)=L \mid \sim p$. As $a \wedge \sim a \leqslant a \vee \sim a, a \wedge \sim a \in g(p)$. We have an impossible situation. Thus,

$$
(a \wedge \sim a)^{\wedge} \cap \bar{X}=\varnothing
$$

for all $a \in L$. Thus, $n(L)$ is contained within the kernel of the congruence $\Theta(\bar{X})$ associated with the closed deceasing set $\bar{X}$. Hence $\Theta(n(L)) \subseteq \Theta(\bar{X})$.

Conversely, let $r, s \in L$ be such that $r \equiv s(\Theta(\bar{X}))$. Then,

$$
\hat{r}=(\hat{r} \cap \bar{X}) \cup(\hat{r} \cap(X \mid \bar{X})) \subseteq s \cup(X \mid \bar{X}) .
$$


Let $p \in X \backslash \bar{X}$. Then $p \nless g(p)$. Thus, there exists $a_{p} \in L$ such $p \in \hat{a}_{p}$ and $g(p) \in X \mid \hat{a}_{p}$ so that $p \in X \mid g\left(\hat{a}_{p}\right)=\left(\sim a_{p}\right)^{\wedge}$. Hence, $\left(a_{p} \wedge \sim a_{p}\right)^{\wedge}$ is a clopen increasing neighbourhood of $p \in X \backslash \bar{X}$. Then, $\{\hat{s}\} \cup\left\{\left(a_{p} \wedge \sim a_{p}\right)^{\wedge}: p \in X \backslash \bar{X}\right\}$ is an open cover of $\hat{r}$. Using compactness, we find that $r \leqslant s \vee\left(a_{1} \wedge \sim a_{1}\right) \vee \ldots \vee\left(a_{n} \wedge \sim a_{n}\right)$ for some suitable $a_{1}, \ldots, a_{n} \in L$. By symmetry, we obtain a similar inequality with the rôles of $r$ and $s$ interchanged. We can now conclude that $r \equiv s(\Theta(n(L)))$. Thus, $\Theta(n(L))=\Theta(\bar{X})$, and (i) is established in view of the remarks which precede this theorem.

The proof of (ii) is dual to that of (i). Then (iii) and (iv) follow from our remarks and 1.3.

Actually 2.2(iii) and 1.3 combine to give an al ternative proof of Theorem 3.2 of Cornish and Fowler (1977)-a result which gives $\mathrm{K}$ and the category Ks of Kleene spaces as dually equivalent categories. In their Theorems 4.4 and 4.5, Berman and Dwinger describe the sets of prime ideals of the quotients $L / \kappa(L)$ and $L / \beta(L)$; our Theorem 2.2 can be regarded as a sharpening of their results.

Perhaps, it should be pointed out that $X_{\mathrm{K}}$ and $X_{\mathrm{B}}$ may be empty. For example, in the case of the tetrad

$$
\{0, a, b, 1: 0=a \wedge b<a, b<a \vee b=1, a=\sim a, b=\sim b\},
$$

they are the dual spaces of the single element Kleene and Boolean algebras, respectively.

Duality together with 2.2 and the results of Section 1 allow us to assert the following corollary. Of course, a direct verification provides the best proof, while 1.3 and 2.2 give the content of the results.

COROLLARY 2.3. Let $(X, g)$ be an object in MS. Then, $\left(X_{\mathrm{K}}, g_{\mathrm{K}}\right)$, with $g_{\mathrm{K}}=g \mid X_{\mathrm{K}}$, is the largest $\mathrm{KS}-$ subobject of $(X, g)$. Furthermore, $\mathrm{KS}$ is a coreflective subcategory of MS and $\left(X_{\mathrm{K}}, g_{\mathrm{K}}\right)$ is the coreflection of $(X, g)$ in $\mathrm{KS}$.

By Theorem 2.4 of Cornish and Fowler (1977), we know that the product $\left(\prod\left(X_{i}, g_{i}\right) ; p_{i}\right)$ of a family $\left\{\left(X_{i}, g_{i}\right)\right\}$ of objects in MS is $\left(\left(\prod X_{i}, \prod g_{i}\right) ; p_{i}\right)$, where $\prod X_{i}$ is the topological product endowed with the direct product order, $\prod g_{i}: \prod X_{i} \rightarrow \prod X_{i}$ is the product function defined by $\left(\prod g_{i}\right)\left(x_{i}\right)=\left(g_{i}\left(x_{i}\right)\right)$, and the $p_{j}$ are just the usual projections of $\prod X_{i}$ onto $X_{j}$, for each $j$.

Category theory (see, for example, Balbes and Dwinger (1974), Theorem 5, p. 29) allows us to assert that the coreflection of the ms-product is the product of the $\mathrm{ks}$ coreflections and so we obtain

THEOREM 2.4. Let $\left\{\left(X_{i}, g_{i}\right)\right\}$ be a family of objects in $\mathrm{Ks}$. Then, $\left(\left(\left(\prod X_{i}\right)_{\mathrm{K}},\left(\prod g_{i}\right)_{\mathrm{K}}\right), q_{i}\right)$, where for each $j, q_{j}$ is the restriction of the projection $p_{j}$ mapping $\prod X_{i}$ onto $X_{j}$, is the Ks-product of the $\left(X_{i}, g_{i}\right)$. Moreover, each of the projections $q_{i}$ is onto.

A method for constructing the coproduct of Kleene algebras follows almost immediately: 
COROLlaRY 2.5. Let $(X, g)$ be the KS-product of a family $\left\{\left(X_{i}, g_{i}\right)\right\}$ of Kleene spaces. Then,

(i) $\vec{X}$ is isomorphic to the TODC-product $\prod \vec{X}_{i}$,

(ii) $\bar{X}$ is isomorphic to the ToDc-product $\prod \bar{X}_{i}$, and

(iii) $X$ is the set-union of $\vec{X}$ and $\bar{X}$.

Before proceeding it might be interesting to point out that Theorem 2.4 was suggested to us by Theorem 1 of Preller (1968); there, Preller considers the nature of the (categorical) product of a family of algebras in an algebraic category which is not closed under the formation of direct products.

As an instance of the use of 2.5 we give the following result which is of independent interest.

THeOREM 2.6 Let $K_{1}, K_{2}$ and $K_{3}$ be Kleene algebras. Then, $K_{1} \coprod_{\mathrm{k}}\left(K_{2} \times K_{3}\right)$ is isomorphic to $\left(K_{1} \coprod_{\mathrm{K}} K_{2}\right) \times\left(K_{1} \coprod_{\mathrm{K}} K_{3}\right)$.

Proof. For $i=1,2,3$ let $\left(X_{i}, g_{i}\right)$ be the dual space of $K_{i}$. Let the dual space of $K_{1} \coprod_{\mathrm{k}}\left(K_{1} \times K_{3}\right)$ be denoted by $(X, g)$.

The dual space of the direct product of two Kleene algebras is isomorphic to the disjoint union of the dual spaces-the topology is the 'disjoint-union topology', the order is the cardinal sum, and the $g$-function is the obvious extension of the two $g$-functions. We will only indicate the details for the underlying Priestley space; the details concerning the compatibility of the isomorphism with the $g$-functions are easy but tedious.

By 2.5

$$
\begin{aligned}
& \vec{X} \cong{ }_{1} \vec{X} \times\left(\vec{X}_{2} \cup \vec{X}_{3}\right)=\left(\vec{X}_{1} \times \vec{X}_{2}\right) \cup\left(\vec{X}_{1} \times \vec{X}_{3}\right) \\
& \overleftarrow{X} \cong\left(\bar{X}_{1} \times \bar{X}_{2}\right) \cup\left(\bar{X}_{1} \times \bar{X}_{3}\right)
\end{aligned}
$$

and

$$
X=\vec{X} \cup \vec{X}
$$

There is no interconnection between the ordered sets $\vec{X}_{1} \times \vec{X}_{2}$ and $\bar{X}_{1} \times \bar{X}_{3}$, nor is there between $\vec{X}_{1} \times \vec{X}_{3}$ and $\bar{X}_{1} \times \bar{X}_{2}$. Thus, it follows that

$$
\left.X \cong\left[\vec{X}_{1} \times \vec{X}_{2}\right) \cup\left(\bar{X}_{1} \times \bar{X}_{2}\right)\right] \cup\left[\left(\vec{X}_{1} \times \vec{X}_{3}\right) \cup\left(\bar{X}_{1} \times \bar{X}_{3}\right)\right] .
$$

Because of 2.5, the above right side is the Priestley space of $\left(K_{1} \coprod_{\mathrm{K}} K_{2}\right) \times\left(K_{1} \coprod_{\mathrm{K}} K_{3}\right)$ and the theorem follows.

Another consequence of 2.4 and 2.5 is the 'subalgebra-coproduct property' for $\mathrm{K}$-coproducts; this was established by purely algebraic means in Theorem 3.8 of Cornish and Fowler (1977) and states that if $A_{1}$ and $B_{1}$ are subalgebras of the Kleene algebras $A$ and $B$, respectively, then $A_{1} \coprod_{\mathrm{K}} B_{1}$ is isomorphic to the subalgebra of $A \coprod_{\mathrm{K}} B$ which is generated by $A_{1}$ and $B_{1}$. 


\section{Free Kleene algebras}

We now turn to the subject of free Kleene algebras. At the end of Section 1, we mentioned that the free De Morgan algebra $F_{M}\left(x_{1}\right)$ and the free Kleene algebra $F_{\mathbf{K}}\left(x_{1}\right)$ on a single free generator $x_{1}$ are one and the same 6-element De Morgan algebra, whose dual space is actually the tetrad $X=\left\{p_{1}, p_{2}, p_{3}, p_{4}: p_{1}<p_{2}, p_{3}<p_{4}\right.$; $p_{2}$ and $p_{3}$ are incomparable; $\left.g\left(p_{1}\right)=p_{4}, g\left(p_{2}\right)=p_{2}, g\left(p_{3}\right)=p_{3}\right\}$, endowed with the discrete topology. Here $\vec{X}=\left\{p_{2}, p_{3}, p_{4}\right\}$ and $\bar{X}=\left\{p_{2}, p_{3}, p_{1}\right\}$. Due to $1.1,1.8$ and 2.5 the dual space of $F_{\mathrm{K}}\left(x_{1}, \ldots, x_{n}\right)$ is $\left(\prod_{1}^{n} \vec{X} \cup \prod_{1}^{n} \bar{X}, g\right)$. As there are $2^{n}$ points in $\left(\prod_{1}^{n} \vec{X}\right) \cap\left(\prod_{1}^{n} \bar{X}\right)$ we have, in view of Corollary 14, p. 73 of Grätzer (1971),

THEOREM 3.1. $F_{\mathbf{K}}\left(x_{1}, \ldots, x_{n}\right)$ is a distributive lattice of length $2.3^{n}-2^{n}$.

Since $\mathrm{K}$ is a variety with distributive congruence lattices and such that each subdirectly irreducible is simple (the only subdirectly irreducibles are 2 and 3), the very general results 1.4 and 1.5 of Berman (1975) can be used to give another proof of 3.1 .

The dual space of $F_{\mathrm{K}}\left(x_{1}, x_{2}\right)$ has 14 elements; it is the subposet of the 16 element poset (lattice) consisting of the direct product of 2 copies of the tetrad obtained by omitting the two points $\left(p_{1}, p_{4}\right)$ and $\left(p_{4}, p_{1}\right)$. Thus, we omit its diagram. However, in Table 3.2 we list its 84 increasing subsets, that is, Table 3.2 gives the list of the 84 elements of free Kleene algebra on two free generators, which are conveniently labelled as $a$ and $b$. The 84 elements are tabulated in 15 rows. These rows are such that each element in a particular row is less than some element in the row above and greater than some element in the row below. Also the rows are such that no two elements in the same row are comparable. The $\sim$-operation works as follows: If $x$ is in the middle row (that is, row 8 ) then $\sim x$ is found in the symmetrical position with respect to the other end of the row. Otherwise, if $x$ is not in the middle row, say $x$ is the $j$ th element in row $i(i \neq 8)$, then $\sim x$ is the $j$ th element in row (16-i).

Free Kleene algebras have also been discussed by Berman and Dwinger (1973) and Berman and Köhler (1976). Amongst other things, Berman and Dwinger gave the length of $F_{\mathrm{K}}\left(x_{1}, \ldots, x_{n}\right)$ and the cardinal of $F_{\mathrm{K}}\left(x_{1}, x_{2}\right)$ while Berman and Köhler have found by means of a general computer program that $F_{\mathrm{K}}\left(x_{1}, x_{2}, x_{3}\right)$ has 43,918 elements. We have retained our Section 3 in the hope that it contains extra information.

\section{TABle 3.2.}

row

11.

$2 a \vee \sim a \vee b \vee \sim b$.

$3 a \vee b \vee \sim b, a \vee \sim a \vee b, a \vee \sim a \vee \sim b, \sim a \vee b \vee \sim b$.

$4 a \vee b \vee(\sim a \wedge \sim b), a \vee \sim b \vee(\sim a \wedge b), a \vee \sim a, b \vee \sim b, \sim a \vee b \vee(a \wedge \sim b)$, $\sim a \vee \sim b \vee(a \wedge b)$ 


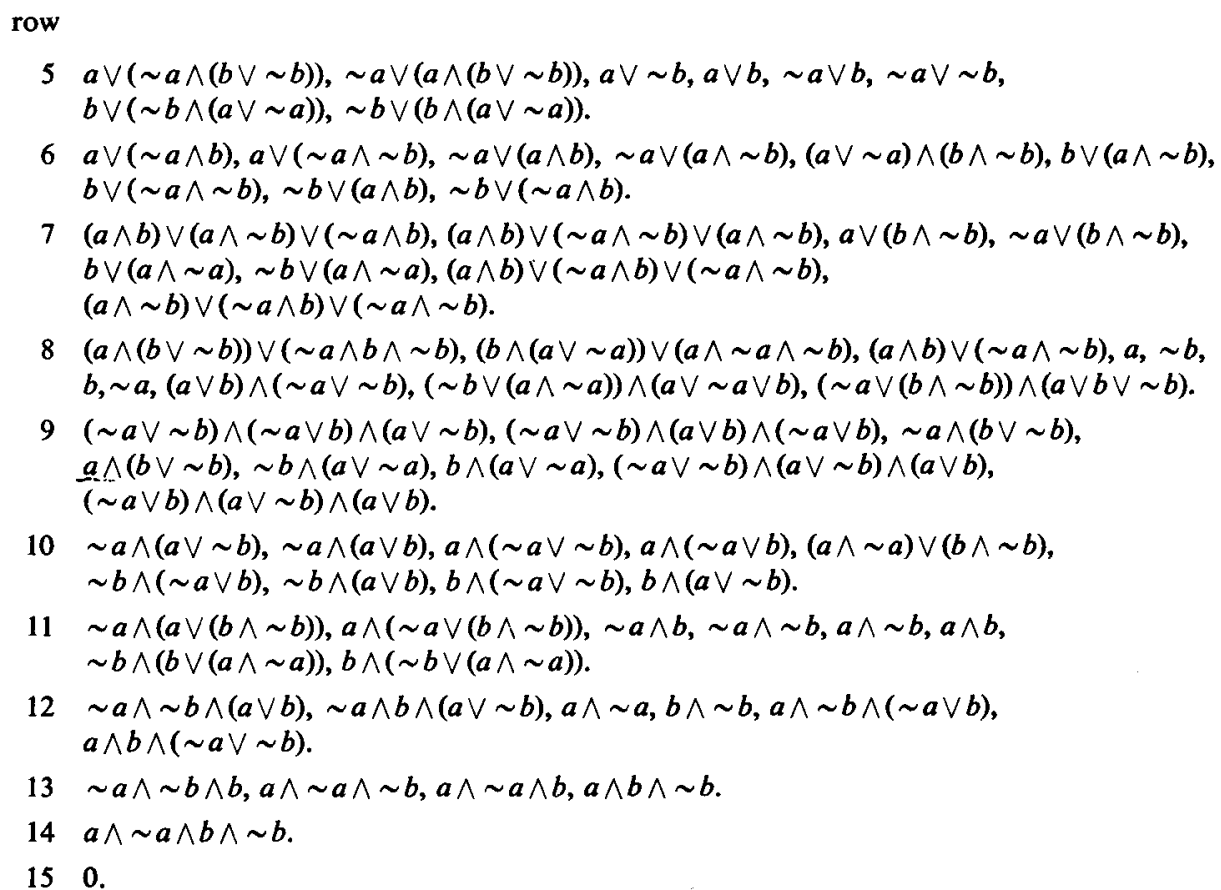

\section{References}

R. Balbes and P. Dwinger (1974), Distributive lattices (University of Missouri Press, Columbia, Missouri).

J. Berman (1975), 'Algebras with modular lattice reducts and simple subdirectly irreducibles', Discrete Math. 11, 1-8.

J. Berman and Ph. Dwinger (1973), 'De Morgan algebras: free products and free algebras', Portugal Math. (submitted).

J. Berman and P. Köhler (1976), 'Cardinalities of finite distributive lattices', Math. Sem. Giessen. 121, $103-124$.

W. H. Cornish (1975), 'On H. Priestley's dual of the category of bounded distributive lattices', Mat. Vesnik. 12, 329-332.

W. H. Cornish and P. R. Fowler (1977), 'Coproducts of De Morgan algebras', Bull. Austral. Math. Soc. 16, 1-13.

G. Grätzer (1971), Lattice theory. First concepts and distributive lattices (W. H. Freeman, San Francisco).

J. Hashimoto (1952), 'Ideal theory for lattices', Math. Japon. 2, 149-186.

J. A. Kalman (1958), 'Lattices with involution', Trans. Amer. Math. Soc. 87, 485-491.

A. Preller (1968), 'On the relationship between the classical and categorical direct product of algebras', Proc. Nederl. Akad. Weterisch. Ser. A 71, 512-516.

A. Urquhart (1978), 'Distributive lattices with a dual homomorphic operation' (to appear).

\section{School of Mathematical Sciences}

The Flinders University of South Australia

Bedford Park, 5042, South Australia 